\title{
Enhanced Catalytic Mechanism of Twin-Structured $\mathrm{BiVO}_{4}$
}

Meirong Huang1, Wanzhen $\mathrm{He}^{2}$, Zhiping $\mathrm{Xu}^{2}$, Hongwei Zhu ${ }^{1}$

${ }^{1}$ State Key Laboratory of New Ceramics and Fine Processing, School of Materials

Science and Engineering, Tsinghua University, Beijing 100084, China

${ }^{2}$ Applied Mechanics Laboratory, Department of Engineering Mechanics, Tsinghua

University, Beijing 100084, China

\section{Methods}

- Calculation methods

- Geometric structure of single-crystal $\mathrm{BiVO}_{4}$

- Geometric structure of twinned $\mathrm{BiVO}_{4}$

- Electronic structure of single-crystal $\mathrm{BiVO}_{4}$

- Gibbs free-energy calculation

- Formation energy of oxygen vacancy

- Visualization and analysis

- Preparation of $\mathrm{BiVO}_{4}$

\section{Figures S1-S15}


Calculation methods: Calculations are performed using the Vienna Ab initio Simulation Package (VASP) which is a density functional theory (DFT) plane wave simulation code. ${ }^{1}$ Exchange-correlation effects are described by Perdew-BurkeErnzerhof (PBE) of generalized gradient approximation (GGA). ${ }^{2}$ Core electrons are treated with a projector augmented wave (PAW) method. ${ }^{3}$ A plane-wave cutoff threshold of $500 \mathrm{eV}$ has enough convergence. Gamma-centered grids have a k-point sampling of $0.04 \AA^{-1}$. For band structure calculation, band energies are plotted along lines between various high-symmetry points (L-M-A- $\Gamma-\mathrm{Z}-\mathrm{V})$ in the Brillouin zone. Here, the high-symmetry points include $\mathrm{L}(-0.5,0,0.5), \mathrm{M}(-0.5,0.5,0.5), \mathrm{A}(-0.5$, $0,0), \Gamma(0,0,0), \mathrm{Z}(0,-0.5,0.5)$, and $\mathrm{V}(0,0,0.5){ }^{1}$

Electronic minimization is performed via a mixture of Davidson and RMM-DIIS (residual minimization scheme/direct inversion in an iterative subspace) methods. The minimization process converges until the total energies and eigenvalues changes between two steps are both smaller than $1 \times 10^{-5} \mathrm{eV}$. Ionic minimization is performed using a conjugate gradient (CG) algorithm which has a step scaling width of $0.1 \AA$. The minimization process converges until the residual average force is less than -0.01 (single crystals) or -0.03 (twinned crystals) $\mathrm{eV} / \AA$. After geometric optimization, static calculations are performed for total energy calculations and electronic structure calculations, including density of states (DOS), band structure, and projected wave functions.

Geometric structure of single-crystal BiVO $\mathrm{B}_{4}: \mathrm{BiVO}_{4}$ has three main crystalline structures, namely monoclinic scheelite, tetragonal zircon, and tetragonal scheelite structure. The monoclinic scheelite structure is commonly used for photocatalysis because of its superior activity. ${ }^{4-5}$ Monoclinic scheelite $\mathrm{BiVO}_{4}$ belongs to space group $15(\mathrm{C} 2 / \mathrm{c})$; its cell constant lengths $a, b, c$ are 7.224, 11.552, $5.108 \AA$, and corresponding angles $\alpha, \beta, \gamma$ are $90^{\circ}, 135^{\circ}, 90^{\circ}$, respectively. Further details about the atomic coordinates of all atoms in $\mathrm{BiVO}_{4}$ are reported in the reference. ${ }^{1}$

Based on these parameters, the primitive cell of $\mathrm{BiVO}_{4}$ is obtained (Fig. S1a). It possesses two kinds of polyhedral, including a $\mathrm{VO}_{4}$ tetrahedron and a $\mathrm{BiO}_{8}$ 
dodecahedron. Namely, $\mathrm{V}$ and $\mathrm{Bi}$ atoms are surrounded by 4 and $8 \mathrm{O}$ atoms, respectively. $\mathrm{A} \mathrm{BiO}_{8}$ dodecahedron connects with a subsequent $\mathrm{BiO}_{8}$ dodecahedron by sharing an edge. $\mathrm{A} \mathrm{BiO}_{8}$ dodecahedron connects with a neighbor $\mathrm{VO}_{4}$ tetrahedron by a shared $\mathrm{O}$ atom. The electronic structure of $\mathrm{BiVO}_{4}$ is calculated using this primitive cell, including band structure and DOS. After geometric optimization, the primitive cell is converted into a unit cell (Fig. S1b). Cell constant lengths $a, b, c$ of the unit cell are 5.192, 5.192, $11.701 \AA$, and corresponding angles $\alpha, \beta, \gamma$ are $90^{\circ}, 90^{\circ}, 90^{\circ}$, respectively.

To perform a study about surface catalysis on $\mathrm{BiVO}_{4}$, the surface is described by a slab in a periodic unit cell. Experimental results and theoretical calculations have shown that the $\mathrm{BiVO}_{4}(001)$ plane has superior photocatalytic activity (crystal indices vary with the definition of cell parameters), ${ }^{6-8}$ a (001)-oriented slab is then cleaved through the optimized unit cell (Fig. S1c). According to the reference, the most stable slab termination exposes 4-fold coordinated $\mathrm{V}$ atoms, 2-fold coordinated $\mathrm{O}$ atoms, and 6-fold coordinated $\mathrm{Bi}$ atoms. ${ }^{9}$ The slab thickness has $6 \mathrm{Bi}$ or $\mathrm{V}$ layers to relieve inevitable surface relaxation. Also, a vacuum thickness of $15 \AA$ is added to the periodic unit cell. The vacuum layer not only keeps the slab far from the influence of its periodic unit cell but also accommodates adsorbates. During surface relaxation, three bottom atomic layers of the slab are fixed to bulk crystal, while the rest layers are allowed to relax. Correction of dipole moment is proceeded to correct the error introduced by periodic boundary conditions. After geometric optimization, a supercell $(2 \times 2 \times 1)$ is built for adsorption calculations, this correspondes to a surface coverage of $1 / 4$ monolayer (ML).

Geometric structure of twinned $\mathrm{BiVO}_{4}$ : Detailed transmission electron microscopy (TEM) characterizations, including high-resolution TEM (HRTEM) images and selected area electron diffraction (SAED) patterns, have been done and analyzed to confirm the twin plane and interfacial atomic configuration of twinned $\mathrm{BiVO}_{4}$. The SAED pattern (zone axis [11ㅣ) indicates that the Miller index of the twin plane is

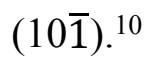


The building process of twinned $\mathrm{BiVO}_{4}$ is shown in Fig. S2. First, to match the crystal structure (Fig. S1b) with the SAED pattern, lattice constants of the crystal structure are redefined through switching $\boldsymbol{b}$ and $\boldsymbol{c}$ axes (Fig. S2a). Second, the redefined unit cell is rebuilt through setting [010], [101], and [101] as $\boldsymbol{a}, \boldsymbol{b}$, and $\boldsymbol{c}$ axes, respectively. As a result, the twin plane is parallel to the ab plane (Fig. S2b) and the zone axis is changed from [1 $\overline{1} 1]$ into [1 $\overline{1} 0]$. Third, the reconstructed unit cell is made symmetrical about the ab plane to construct twinned strucutres (Fig. S2c). After that, the HRTEM image is used to describe the interfacial atomic configuration of twin boundaries where no periodic $\mathrm{Bi}$ or $\mathrm{V}$ vacancies are found in the coherent twin boundaries. Therefore, among six proposed interfacial atomic configurations, those two without Bi or $\mathrm{V}$ vacancies are more likely to exist stably (Fig. S3). Because of their similarity, the following discussion mainly focuses on one of them.

The optimized unit cell of twinned $\mathrm{BiVO}_{4}$ is shown in Figs. S4a, b, only a length of $23 \mathrm{Bi}$ or $\mathrm{V}$ layers (perpendicular to twin boundaries) is considered to reduce computational costs, while there are still $176 \mathrm{O}, 44 \mathrm{Bi}$, and $44 \mathrm{~V}$ atoms. For surface catalysis, a slab with (100) plane (equivalent to the (001) plane of single-crystal $\mathrm{BiVO}_{4}$ ) is cleaved through the optimized unit cell (Fig. S4c). Surface termination, atomic layer thickness, and vacuum thickness are the same as that of single crystals. Since the interactions between adsorbates on $\mathrm{BiVO}_{4}$ along the $-\mathrm{O}-\mathrm{Bi}-\mathrm{O}-\mathrm{V}-$ direction are not significantly affected if surface coverage is less than or equal to $1 / 4 \mathrm{ML}$, the optimized slab is directly used for calculation of the single-site adsorption.

Electronic structure of single-crystal $\mathrm{BiVO}_{4}$ : $\mathrm{DOS}$ and band structure of $\mathrm{BiVO}_{4}$ are calculated using the primitive cell. Valence band maximum (VBM) is defined as the Fermi level, which is moved to the zero of energy. According to the partial DOS (PDOS) (Fig. S9), the lower valence band (VB) is dominated by Bi 6s orbitals and slightly hybridized with O 2 p orbitals. Upper VB and lower conduction band (CB) are mainly composed of O 2p, Bi 6p, and V 3d orbitals. Small contributions of Bi 6s orbitals are also observed in regions near the Fermi level. 
To learn more about the chemical bonding features of $\mathrm{BiVO}_{4}$, the projected wave functions in different energy ranges are analyzed and visualized in Fig. S10. These energy partitions are divided through the curvilinear shape. Bi 6s, Bi 6p, or V 3d orbitals hybridize with $\mathrm{O} 2 \mathrm{p}$ orbitals to form bonding states in energy regions E1 E5. Antibonding features are mainly observed in energy regions E8 E10. These antibonding states are derived from the hybridization of Bi $6 \mathrm{p}$ or $\mathrm{V} 3 \mathrm{~d}$ with $\mathrm{O} 2 \mathrm{p}$ orbitals. The energy region near VBM (E6) is dominated by non-bonding states derived from Bi $6 s$ and $\mathrm{O} 2 \mathrm{p}$ orbitals. Stereochemical active Bi $6 \mathrm{~s}$ lone pairs are generated owing to the lower symmetry of scheelite $\mathrm{BiVO}_{4}$ structure according to the second-order Jahn-Teller (SOTJ) distortion. ${ }^{11}$ Non-bonding states of O $2 p$ are beneficial for a direct interaction between lattice oxygen $\left(\mathrm{O}_{\text {lat }}\right)$ and adsorbed oxygen $\left(\mathrm{O}_{\mathrm{ads}}\right)$ atoms. While in the region near $\mathrm{CB}$ minimum $(\mathrm{CBM})$, namely region $\mathrm{E} 7$ is composed of non-bonding states arising from V 3d orbitals. Detailed bonding features have been discussed in the related reference. ${ }^{1}$ The proposed molecular-orbital bonding diagram is illustrated in Fig. S11. No antibonding states are observed below the Fermi level, which is favorable for stabilizing $\mathrm{O}_{\text {lat }}$ atoms in $\mathrm{BiVO}_{4}$. Thus, it can be predicted that the formation of oxygen vacancies in $\mathrm{BiVO}_{4}$ is difficult.

The calculated band structure of $\mathrm{BiVO}_{4}$ along a high-symmetry line (L-M-A- $\Gamma-\mathrm{Z}-$ V) is shown in Fig. S6. VBM and CBM locate at different k-points, so $\mathrm{BiVO}_{4}$ is an indirect bandgap semiconductor which has a bandgap of $2.11 \mathrm{eV}$. This bandgap is smaller than its experimental value $(2.4 \mathrm{eV})$ due to the well-known shortcoming of DFT. However, three direct transitions are observed at $\mathrm{L}, \mathrm{A}$, and $\mathrm{Z}$ points, possessing a bandgap of about $2.18 \mathrm{eV}$. Since they are slightly larger than the indirect bandgap, $\mathrm{BiVO}_{4}$ can be regarded as a direct bandgap semiconductor. These results coincide well with the associated references. ${ }^{1,12}$ The ideal bandgap of $\mathrm{BiVO}_{4}$ can be explained through its molecular-orbital bonding diagram. The hybridization between $\mathrm{Bi} 6 \mathrm{~s}$ and $\mathrm{O}$ $2 \mathrm{p}$ forces an upward dispersion of VB. The hybridization between V $3 \mathrm{~d}, \mathrm{O} 2 \mathrm{p}$, and Bi $6 \mathrm{p}$ leads to a downward of $\mathrm{CB}$. As a consequence, $\mathrm{BiVO}_{4}$ has a visible light-responsive bandgap. ${ }^{12-13}$ 
Gibbs free-energy calculation: Traditional adsorbate evolution mechanism $\left(\mathrm{AEM}_{\mathrm{t}}\right)$ is typically assumed to involve four concerted proton-electron transfer (CPET) reaction steps and one $\mathrm{O}_{2}$ desorption step centered on metal sites. As described in Eqs. (1-5), one $\mathrm{OH}^{-}$is consumed at each CPET step, and one electron is simultaneously injected into the counter electrode. In detail, one $\mathrm{OH}^{-}$first adsorbs on the slab. Next, the adsorbed $\mathrm{OH}\left(\mathrm{HO}^{*}, \mathrm{~A}_{\mathrm{t} 2}\right)$ undergoes deprotonation to form $\mathrm{O} *\left(\mathrm{~A}_{\mathrm{t} 3}\right)$. Then, the $\mathrm{O} *$ reacts with another $\mathrm{OH}^{-}$to form $\mathrm{HOO}^{*}\left(\mathrm{~A}_{t 4}\right)$. Finally, the OO* state $\left(\mathrm{A}_{\mathrm{t} 5}\right)$ is formed through a deprotonation process, and then $\mathrm{O}_{2}$ is released spontaneously to restore the reactive site of the $\operatorname{slab}\left(*, \mathrm{~A}_{\mathrm{t} 1}\right) \cdot{ }^{14}$

$$
\begin{gathered}
*+\mathrm{OH}^{-} \rightarrow \mathrm{HO} *+\mathrm{e}^{-} \\
\mathrm{HO} *+\mathrm{OH}^{-} \rightarrow \mathrm{O} *+\mathrm{e}^{-}+\mathrm{H}_{2} \mathrm{O} \\
\mathrm{O} *+\mathrm{OH}^{-} \rightarrow \mathrm{HOO} *+\mathrm{e}^{-} \\
\mathrm{HOO} *+\mathrm{OH}^{-} \rightarrow \mathrm{OO} *+\mathrm{e}^{-}+\mathrm{H}_{2} \mathrm{O} \\
\mathrm{OO} * \rightarrow *+\mathrm{O}_{2}(\mathrm{~g})
\end{gathered}
$$

Reaction free energies $(\Delta G)$ for the reaction steps are calculated by Eqs. (6-10), where $G\left(\mathrm{H}^{+}+\mathrm{e}^{-}\right)$is equal to $\frac{1}{2} G_{\mathrm{H}_{2}}$ according to the definition of standard hydrogen electrode (SHE), and the external bias $(U)$ acts on reaction free energies by the $e U$ term. For photocatalysis, the external bias is a summation of equilibrium potential $\left(E^{0}\right)$ and OER overpotential $\left(U_{\mathrm{OER}}\right)$. Here, the overpotential is replaced by the onset potential. Therefore, the $e U$ term $(2.1 \mathrm{eV})$ is obtained by Eq. (11), where $E_{\mathrm{g}}$ is the bandgap of $\mathrm{BiVO}_{4}(2.4 \mathrm{eV})$ and $E_{\mathrm{v}}$ is the externally applied electrical energy ( $0.3 \mathrm{eV}){ }^{6}, 15$

$$
\begin{gathered}
\Delta G_{1}=G_{\mathrm{HO} *}+\frac{1}{2} G_{\mathrm{H}_{2}}-G_{*}-G_{\mathrm{H}_{2} \mathrm{O}}-e U \\
\Delta G_{2}=G_{\mathrm{O} *}+\frac{1}{2} G_{\mathrm{H}_{2}}-G_{\mathrm{HO} *}-e U \\
\Delta G_{3}=G_{\mathrm{HOO} *}+\frac{1}{2} G_{\mathrm{H}_{2}}-G_{\mathrm{O} *}-G_{\mathrm{H}_{2} \mathrm{O}}-e U \\
\Delta G_{4}=G_{\mathrm{OO} *}+\frac{1}{2} G_{\mathrm{H}_{2}}-G_{\mathrm{HOO} *}-e U \\
\Delta G_{5}=G_{*}+G_{\mathrm{O}_{2}}-G_{\mathrm{OO} *}
\end{gathered}
$$




$$
e U=\mathrm{e}\left|E^{0}+U_{\mathrm{OER}}\right|=E_{\mathrm{g}}+E_{\mathrm{v}}
$$

The most positive value among $\Delta G_{1}, \Delta G_{2}, \Delta G_{3}, \Delta G_{4}$, and $\Delta G_{5}$ determines the OER overpotential. The reaction energy of each elementary step is directly available from DFT-calculated total energy. These energies are calibrated with thermal correction at $298 \mathrm{~K}$ to obtain corresponding Gibbs free energies.

Formation energy of oxygen vacancy: For neutral vacancy, the formation energy of oxygen vacancy $\left(E_{\mathrm{O}_{\mathrm{V}}}\right)$ is related to system energy of defective crystal $\left(E_{\mathrm{d}}\right)$, system energy of perfect crystal $\left(E_{\mathrm{p}}\right)$, and oxygen chemical potential $\left(\mu_{0}\right)$, according to Eq. (12). Under an oxidation limit (O-rich limit) condition, $\mu_{0}$ is given by $\frac{1}{2} E_{\mathrm{O}_{2}} \cdot{ }^{16}$

$$
E_{\mathrm{V}_{\mathrm{o}}}=E_{\mathrm{d}}-E_{\mathrm{p}}+\mu_{\mathrm{O}}
$$

Visualization and analysis: Visualization of charge density and geometric structure is performed using VESTA. ${ }^{17}$ To get further insight into the nature of adsorbate-surface interactions, the cooperative orbital Hamiltonian overlap (COHP) is used to analyze DFT-calculated electronic structure. ${ }^{18} \mathrm{COHP}$ provides a quantitative measure of bond strength. Here, positive and negative COHP values represent bonding and antibonding interactions, respectively.

Preparation of $\mathrm{BiVO}_{4}: \mathrm{BiVO}_{4}$ films are prepared by the electrodeposition and annealing $\left(500{ }^{\circ} \mathrm{C}\right)$ methods. Single-crystal $\mathrm{BiVO}_{4}$ is electrodeposited at $-70 \mathrm{mV}$ with a deposition time of $960 \mathrm{~s}$, while the electrodeposition voltage of twin-crystal $\mathrm{BiVO}_{4}$ is $-10 \mathrm{mV}$. Detailed preparation methods are shown in the Experimental Section in ref. 10.

\section{References}

(1) Zhao, Z.; Li, Z.; Zou, Z. Electronic Structure and Optical Properties of Monoclinic Clinobisvanite $\mathrm{BiVO}_{4}$. Phys. Chem. Chem. Phys. 2011, 13, 4746-4753.

(2) Perdew, J. P.; Burke, K.; Ernzerhof, M. Generalized Gradient Approximation Made Simple. Phys. Rev. Lett. 1996, 77, 3865-3868. 
(3) Blochl, P. E. Projector Augmented-Wave Method. Phys. Rev. B 1994, 50, $17953-$ 17979.

(4) Zhao, Y.; Li, R.; Mu, L.; Li, C. Significance of Crystal Morphology Controlling in Semiconductor Based Photocatalysis: A Case Study on $\mathrm{BiVO}_{4}$ Photocatalyst. Cryst. Growth Des. 2017, 17, 2923-2928.

(5) Ding, K.; Chen, B.; Fang, Z.; Zhang, Y. Density Functional Theory Study on the Electronic and Optical Properties of Three Crystalline Phases of $\mathrm{BiVO}_{4}$. Theor. Chem. Acc. 2013, 132, 1352.

(6) Yang, J.; Wang, D.; Zhou, X.; Li, C. A Theoretical Study on the Mechanism of Photocatalytic Oxygen Evolution on $\mathrm{BiVO}_{4}$ in Aqueous Solution. Chem. Eur. J. 2013, 19, 1320-1326.

(7) Han, H. S.; Shin, S.; Kim, D. H.; Park, I. J.; Kim, J. S.; Huang, P.-S.; Lee, J.-K.; Cho, I. S.; Zheng, X. Boosting the Solar Water Oxidation Performance of a BiVO Photoanode by Crystallographic Orientation Control. Energy Environ. Sci. 2018, 11, 1299.

(8) Xi, G.; Ye, J. Synthesis of Bismuth Vanadate Nanoplates with Exposed $\{001\}$ Facets and Enhanced Visible-Light Photocatalytic Properties. Chem. Commun. 2010, 46, 1893-1895.

(9) Zhao, Z.; Li, Z.; Zou, Z. Structure and Energetics of Low-Index Stoichiometric Monoclinic Clinobisvanite $\mathrm{BiVO}_{4}$ Surfaces. RSC Adv. 2011, 1, 874-883.

(10) Huang, M.; Li, C.; Zhang, L.; Chen, Q.; Zhen, Z.; Li, Z.; Zhu, H. Twin Structure in $\mathrm{BiVO}_{4}$ Photoanodes Boosting Water Oxidation Performance Through Enhanced Charge Separation and Transport. Adv. Energy Mater. 2018, 8, 1802198.

(11) Stoltzfus, M. W.; Woodward, P. M.; Seshadri, R.; Klepeis, J.-H.; Bursten, B. Structure and Bonding in $\mathrm{SnWO}_{4}, \mathrm{PbWO}_{4}$, and $\mathrm{BiVO}_{4}$ : Lone Pairs vs Inert Pairs. Inorg. Chem. 2007, 46, 3839-3850.

(12) Walsh, A.; Yan, Y.; Huda, M. N.; Al-Jassim, M. M.; Wei, S.-H. Band Edge Electronic Structure of $\mathrm{BiVO}_{4}$ : Elucidating the Role of the Bi s and V d Orbitals. Chem. of Mater. 2009, 21, 547-551.

(13) Dolgos, M. R.; Paraskos, A. M.; Stoltzfus, M. W.; Yarnell, S. C.; Woodward, P. M. The Electronic Structures of Vanadate Salts: Cation Substitution as a Tool for Band Gap Manipulation. J. Solid State Chem. 2009, 182, 1964-1971.

(14) Song, J.; Wei, C.; Huang, Z.-F.; Liu, C.; Zeng, L.; Wang, X.; Xu, Z. J. A Review on Fundamentals for Designing Oxygen Evolution Electrocatalysts. Chem. Soc. Rev. 2020, 49, 2196-2214.

(15) Hu, J.; Zhao, X.; Chen, W.; Su, H.; Chen, Z. Theoretical Insight into the Mechanism of Photoelectrochemical Oxygen Evolution Reaction on $\mathrm{BiVO}_{4}$ Anode with Oxygen Vacancy. J. Phys. Chem. C 2017, 121, 18702-18709.

(16) Tanaka, I.; Oba, F.; Tatsumi, K.; Kunisu, M.; Nakano, M.; Adachi, H. Theoretical Formation Energy of Oxygen-Vacancies in Oxides. Mater. Trans. 2002, 43, 1426-1429. (17) Momma, K.; Izumi, F. VESTA: A Three-Dimensional Visualization System for Electronic and Structural Analysis. J. Appl. Crystallogr. 2008, 41, 653-658.

(18) Nelson, R.; Ertural, C.; George, J.; Deringer, V. L.; Hautier, G.; Dronskowski, R. LOBSTER: Local Orbital Projections, Atomic Charges, and Chemical-Bonding 
Analysis from Projector-Augmented-Wavebased Density-Functional Theory. $J$. Comput. Chem. 2020, 41, 1-10.

(19) Bard, A. J.; Bocarsly, A. B.; Fan, F.-R. F.; Walton, E. G.; Wrighton, M. S. Concept of Fermi Level Pinning at Semiconductor/Liquid Junctions. Consequences for Energy Conversion Efficiency and Selection of Useful Solution Redox Couples in Solar Devices. J. Am. Chem. Soc. 1980, 102, 3671-3677. 


\section{Supplementary Figures}
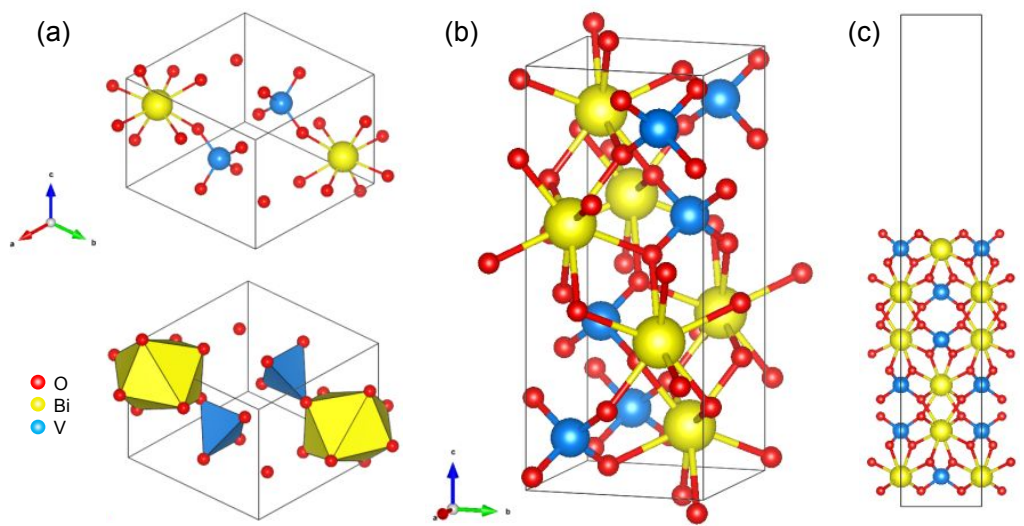

Figure S1. Geometric structure of BiVO $\mathbf{4}_{\text {• }}$ (a) The primitive cell. (b) The unit cell. (c) The (001) slab model cleaved from the unit cell.
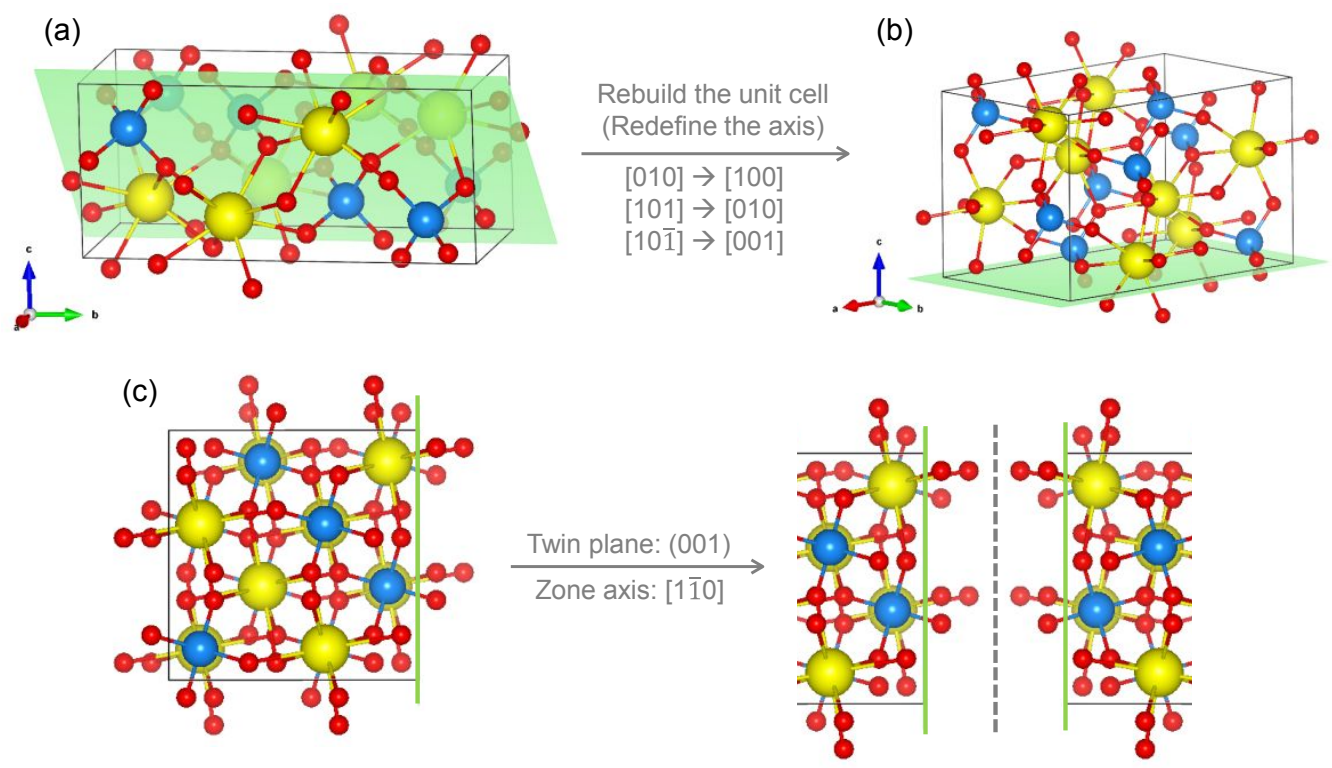

Figure S2. Building process of twinned $\mathbf{B i V O}_{4}$ • (a) The redefined unit cell. (b) The reconstructed unit cell. (c) Construction of twinned structures. Green planes represent the twin planes. 
(a)

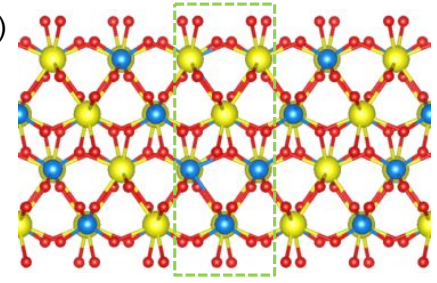

(b)

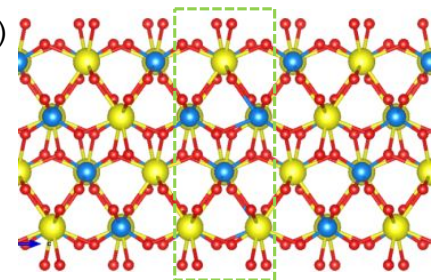

(c)

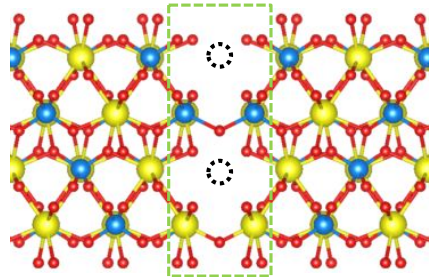

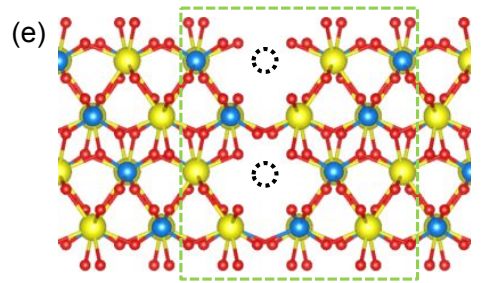

(d)

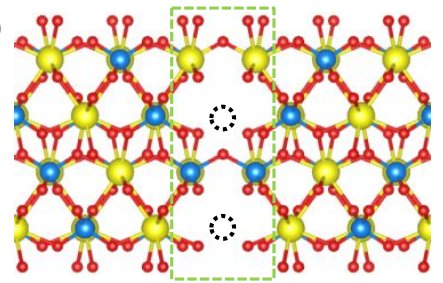

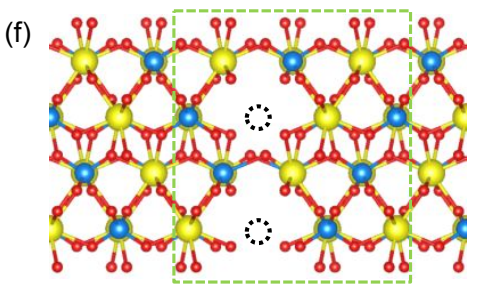

Figure S3. Six possible interfacial atomic configurations of twin boundaries in BiVO $_{4}$. Black dashed circles represent Bi or V vacancies. Green dashed boxes indicate the positions of twin boundaries.

(a)

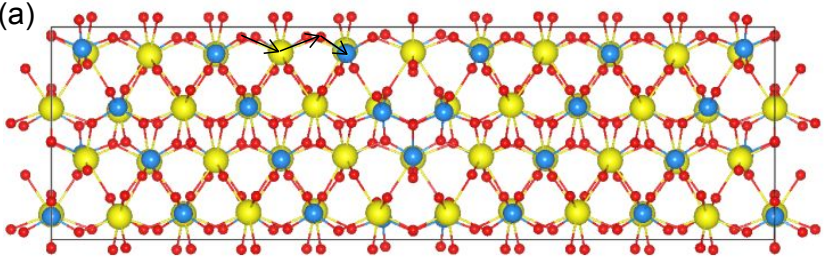

(b)

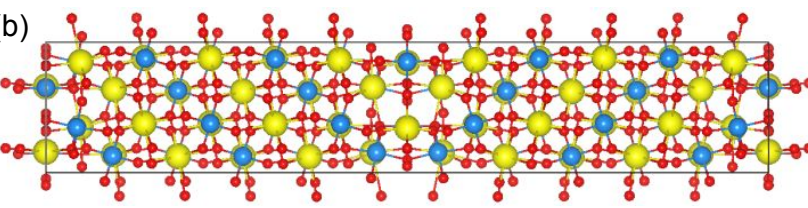

(c)

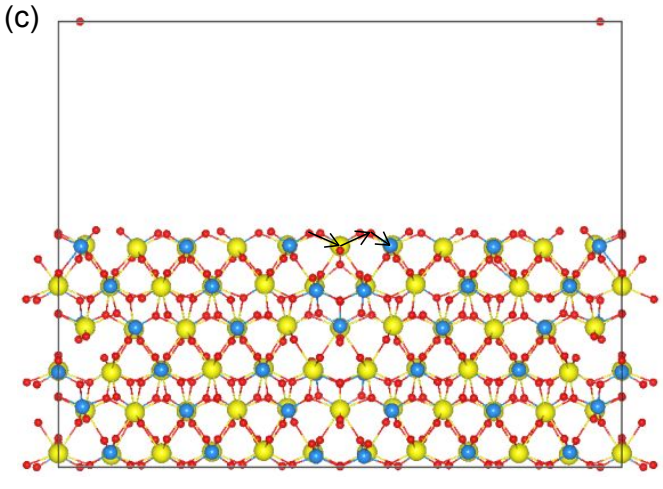

Figure S4. Optimized geometric structure of twinned $\mathbf{B i V O}_{4} \cdot(\mathrm{a}, \mathrm{b})$ The unit cell viewed from different perspectives. (c) The (100) slab model cleaved from the unit cell. Black arrows represent the -O-Bi-V-O- direction. 

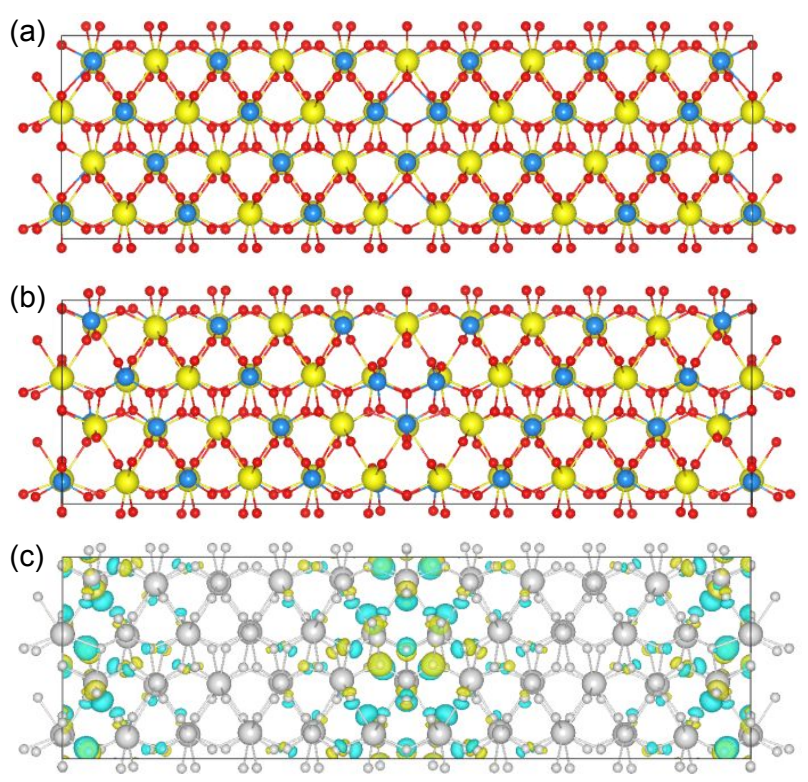

Figure S5. Geometric structure and DCD diagram of twinned $\mathrm{BiVO}_{4}$. (a) The constructed geometric structure. (b) The optimized geometric structure. (c) The DCD diagram.

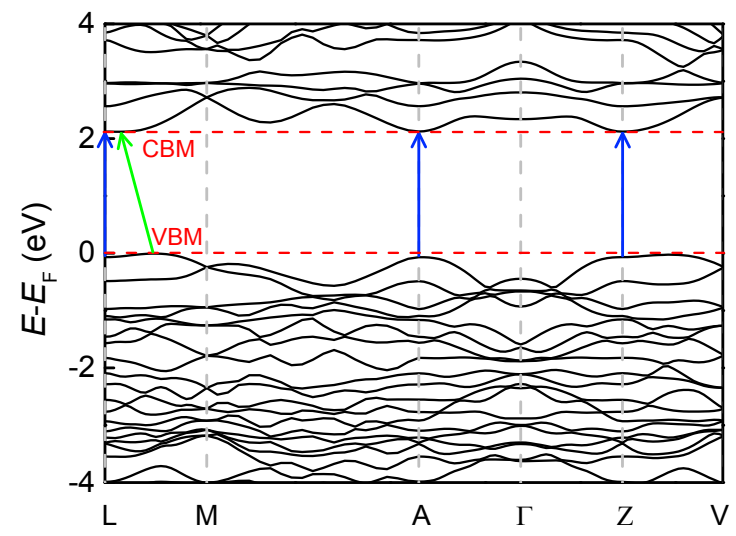

Figure S6. Band structure of $\mathbf{B i V O}_{4}$. Green and blue arrows represent direct and indirect transitions, respectively.
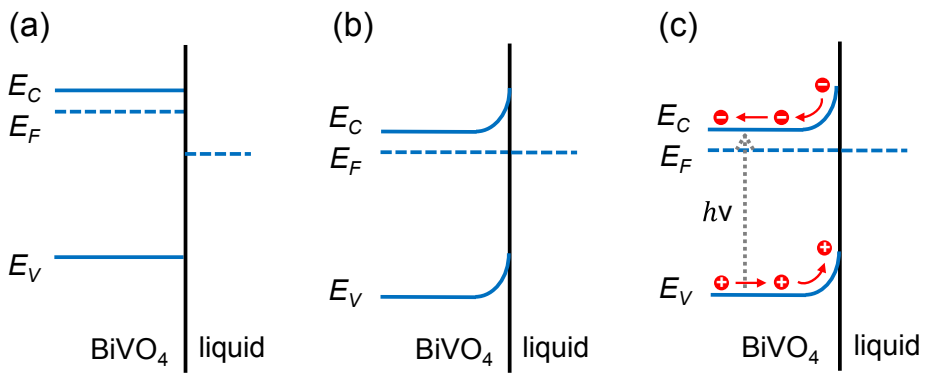

Figure S7. Band energetics of a $\mathrm{BiVO}_{4} /$ liquid junction shown in three cases. ${ }^{19}$ (a) Before equilibrium in the dark. (b) After equilibrium in the dark. (c) Quasi-equilibrium under light illumination. 

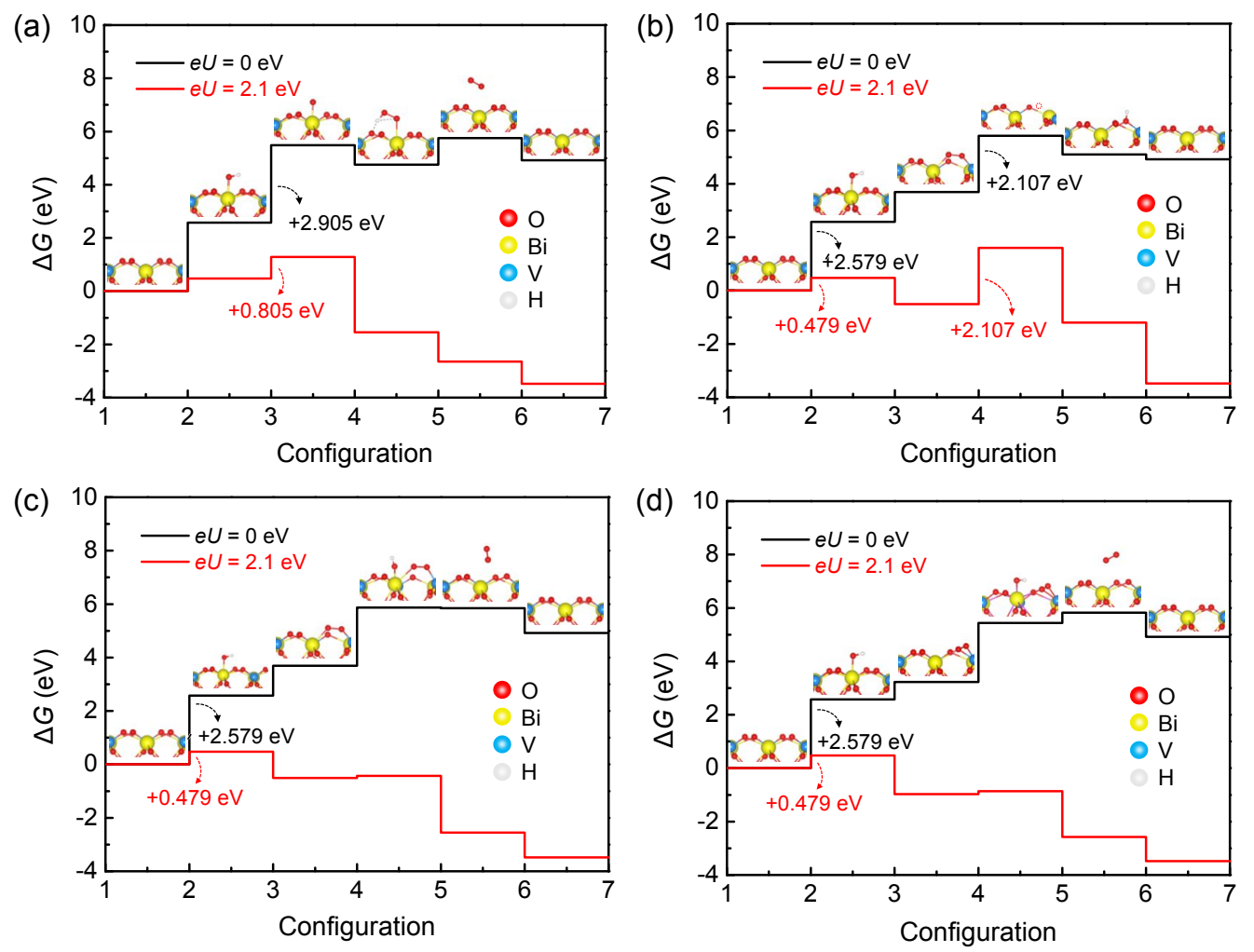

Figure S8. Free energy profiles and corresponding local configurations of four proposed OER mechanisms on $\mathrm{BiVO}_{4}$ slabs. (a) $\mathrm{AEM}_{\mathrm{t}}$ (b) $\mathrm{LOM}_{t}$ (the red dashed circle represents an oxygen vacancy). (c) $\mathrm{AEM}_{\mathrm{s}}$. (d) $\mathrm{LOM}_{\mathrm{s}}$.

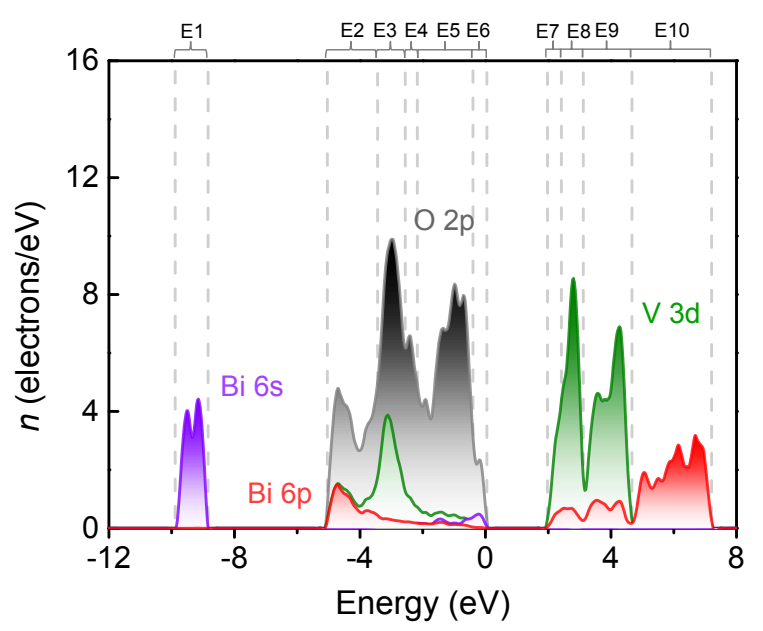

Figure S9. PDOS of $\mathrm{BiVO}_{4}$. Gray dotted lines divide different energy ranges (E1 E10). 


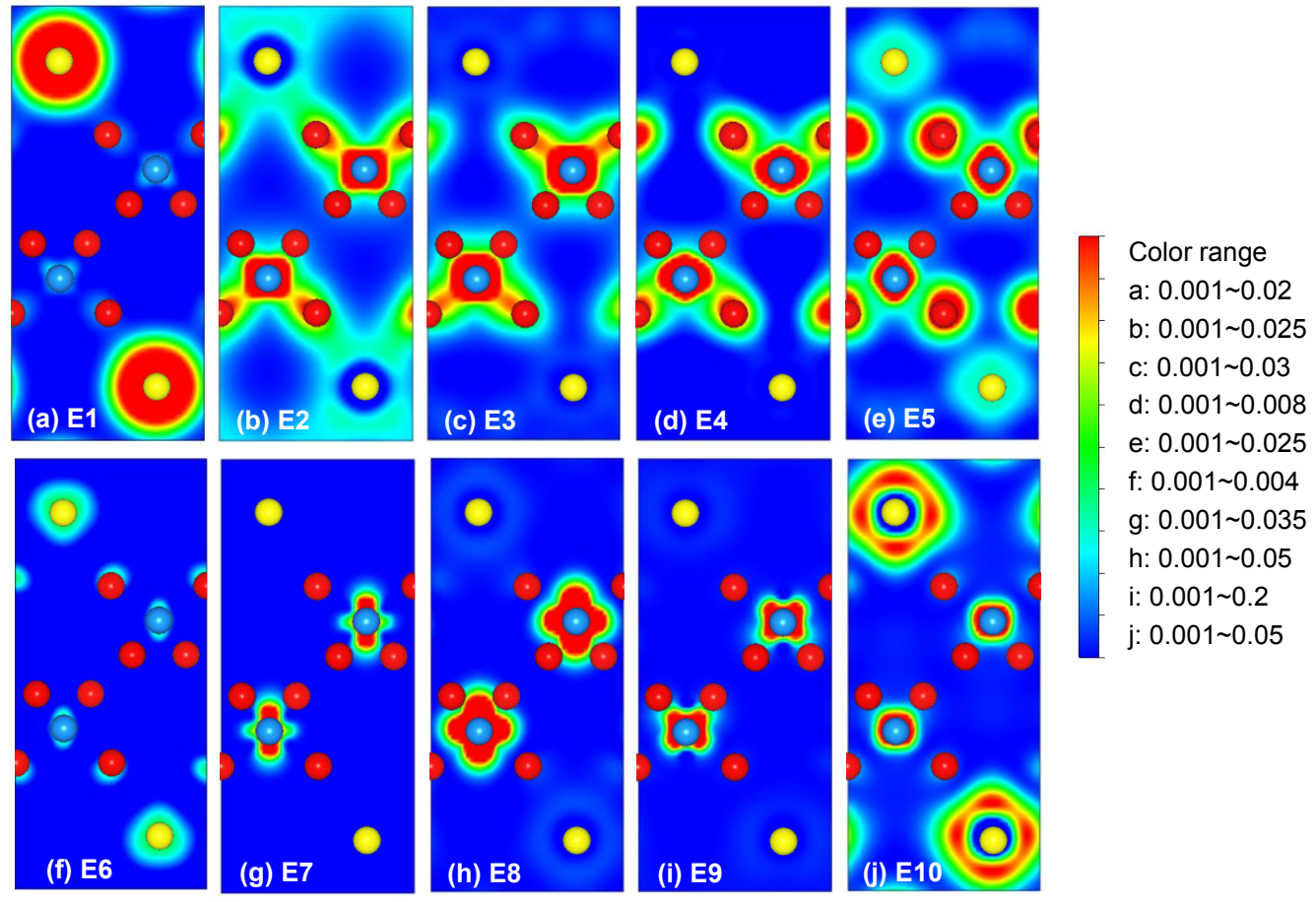

Figure S10. Projected wave functions contour plots through the (100) plane of the unit cell.

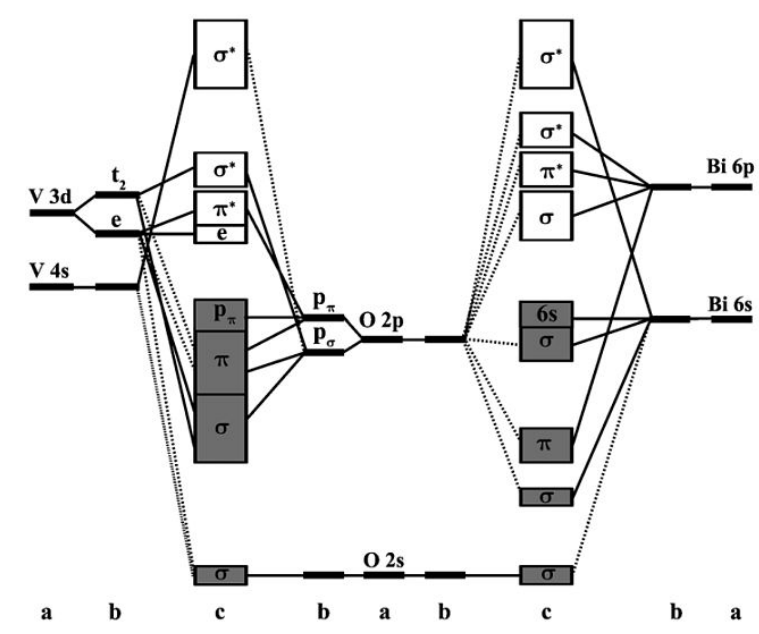

Figure S11. Molecular-orbital bonding diagram of $\mathbf{B i V O}_{4} \cdot{ }^{1}$ Here, a, b, and c represent atomic level, crystal-field split level, and finial interaction states, respectively. The solid and dashed lines represent large and small contributions, respectively. 


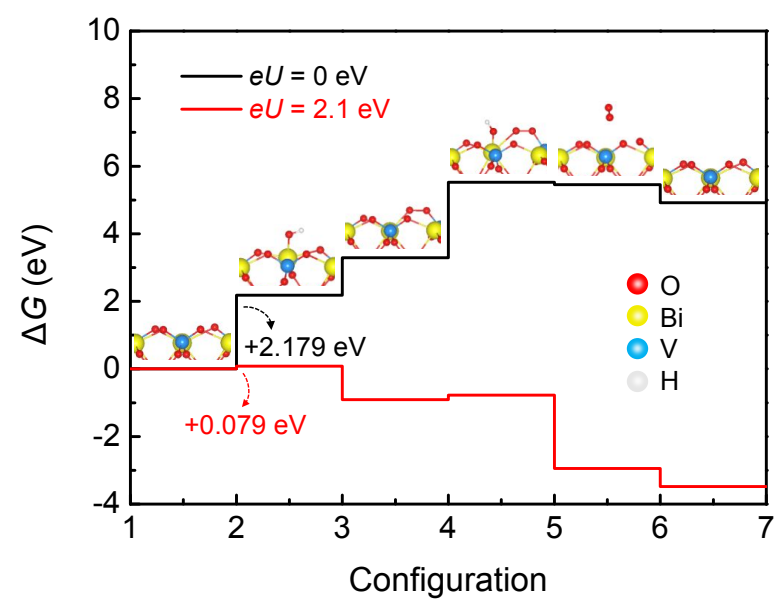

Figure S12. Free energy profiles and corresponding local configurations of the $\mathrm{AEM}_{\mathrm{s}}$ mechanism on the slab of twinned $\mathrm{BiVO}_{4}$.
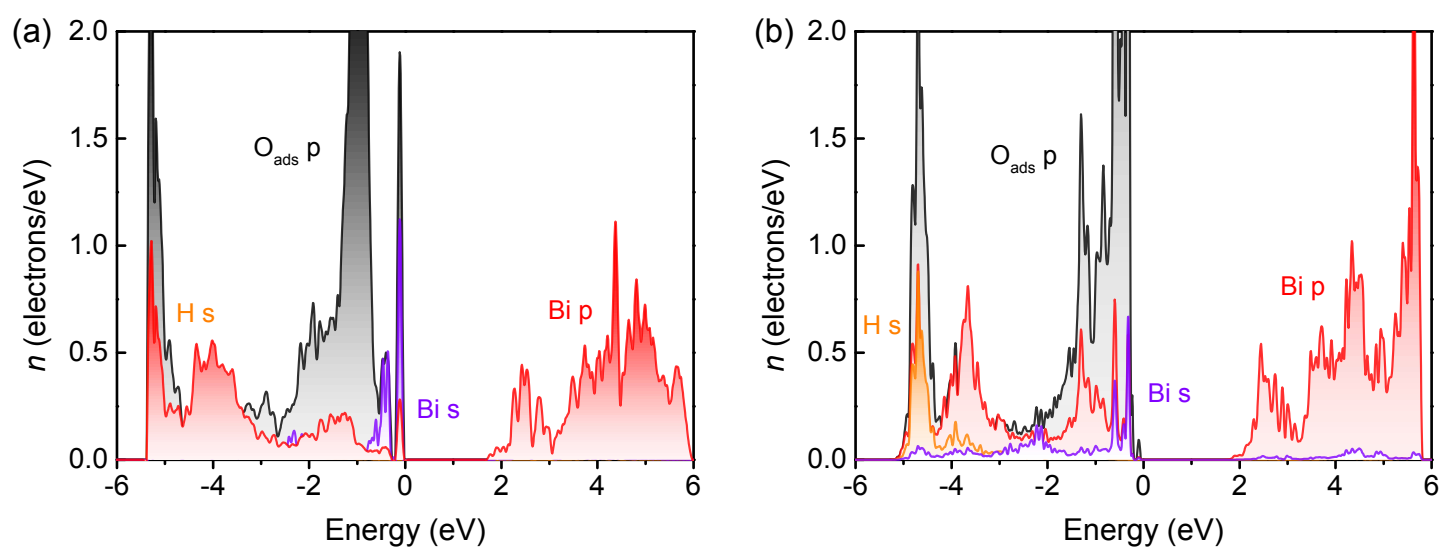

Figure S13. PDOS of $\mathrm{HO}$ adsorbates and $\mathrm{Bi}$ adsorption sites on $\mathrm{BiVO}_{4}$ slabs. (a) Single-crystal $\mathrm{BiVO}_{4}$. (b) Twinned-crystal $\mathrm{BiVO}_{4}$.
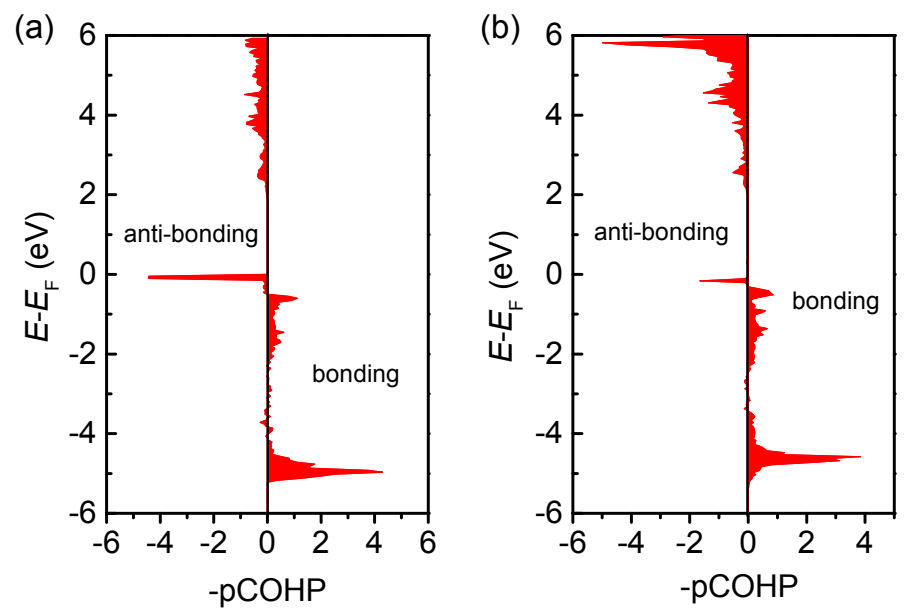

Figure S14. COHP of the $\mathrm{O}_{\text {ads }}-\mathrm{Bi}$ bonds formed between $\mathrm{HO}$ adsorbates and $\mathrm{Bi}$ adsorption sites on $\mathrm{BiVO}_{4}$ slabs. (a) Single-crystal $\mathrm{BiVO}_{4}$. (b) Twinned-crystal $\mathrm{BiVO}_{4}$. 
(a)

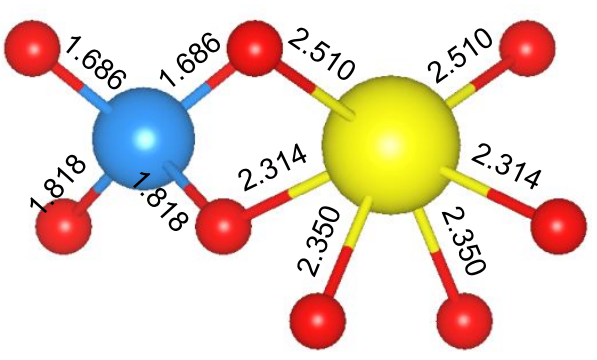

(b)

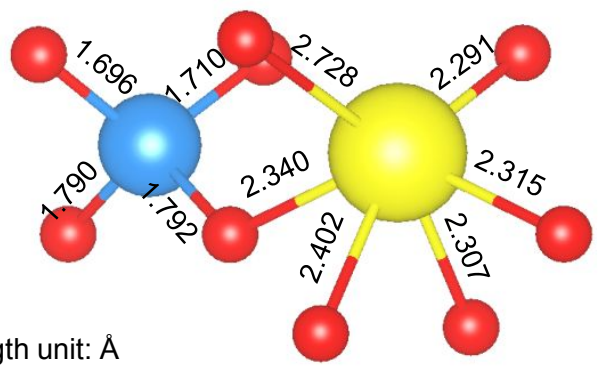

Figure S15. Local atomic configurations of the adsorption sites on $\mathrm{BiVO}_{4}$ slabs. (a) Single-crystal $\mathrm{BiVO}_{4}$. (b) Twinned-crystal $\mathrm{BiVO}_{4}$. 